\title{
The incidence and features of serological $A B O$ subgroups among one million blood donors in Beijing
}

\author{
Zhiyuan $\mathrm{Xu}^{1 \Delta}$, Yushiang $\mathrm{Lin}^{2 \Delta}$, Xiaofei $\mathrm{Li}^{1}$, Haochun Chang ${ }^{2}$, Daowang Fan ${ }^{1}$, Yan Qiu ${ }^{1}$, Tianhong Miao ${ }^{1 *}$ \\ ${ }^{1}$ Blood Group Lab, Beijing Red Cross Blood Center, Beijing 100088, China; \\ ${ }^{2}$ Department of Clinical Medicine, School of Basic Medical Sciences, Peking University Health Science Center, Beijing 100191, China
}

\begin{abstract}
This study aims to determine the incidence of serological ABO subgroups from a large-scale database, along with the features of blood samples with serological ABO discrepancies. The serological ABO results of one million individuals were randomly sampled from a blood donor database in Beijing between 2009 and 2010. All samples were diagnosed by serological reverse and forward $\mathrm{ABO}$ typing using an automatic analyzer. The proportions of the normal $\mathrm{ABO}$ types were $27.28 \%, 31.57 \%, 30.56 \%$, and $10.16 \%$ for blood types $\mathrm{A}, \mathrm{B}, \mathrm{O}$, and $\mathrm{AB}$, respectively. In samples in which $\mathrm{ABO}$ discrepancies or obvious weak agglutinin were identified in the forward or reverse typing, further tests to analyze the $\mathrm{ABO}$ subgroup were conducted. The overall incidence of $\mathrm{ABO}$ subgroups was $0.047 \%$, with 14 ABO subgroups observed: A2, A3, Ax, Am, Aint, Aend, B2, B3, Bx, Bm, Bel, B(A), cis $\mathrm{AB}$, and $\mathrm{AB}^{\mathrm{h}}$. In conclusion, this study revealed the exact normal $\mathrm{ABO}$ and subgroup distributions in the general, healthy population of Beijing using samples from a blood donor database.
\end{abstract}

Keywords: $\mathrm{ABO}$ subtypes, $\mathrm{ABO}$ discrepancy, blood donor, large-scale study

\section{INTRODUCTION}

Accurate serological typing for $\mathrm{ABO}$ types is the primary and most important test in clinical transfusion medicine, because the development of $\mathrm{ABO}$ antigenantibody complexes can induce severe acute or delayed hemolytic reactions ${ }^{[1.2]}$. The distributions of the $\mathrm{ABO}$ blood types and predilections of $\mathrm{ABO}$ subgroups show various incidences among different ethnicities and even among people from the same country but in different areas. For instance, para-Bombay is the most common ABO subgroup in southwestern Asia, whereas it has a very low frequency in northern Chi$\mathrm{na}^{[3,4]}$. Therefore, exact data on the $\mathrm{ABO}$ distribution

\footnotetext{
*Correspondence to: Tianhong Miao, Blood Group Lab, Beijing Red Cross Blood Center, Beijing 100088, China. TEL: +86-10-82807272; E-mail: tianhongm@163.com.

${ }^{ \pm}$These authors contributed equally to this work.
}

and frequencies of the $\mathrm{ABO}$ subgroups are necessary for establishing national transfusion and transplant policies and for offering a reference to create strategies related to blood transfusion and serological typing in each country.

Several case reports focusing on $\mathrm{ABO}$ subgroups have been published, whereas large-scale studies on the incidence of $\mathrm{ABO}$ subgroups in the normal population are limited ${ }^{[5,6]}$. However, there are certain factors that need to be considered, including unexpected antibody interference, and diseases causing weak serological $\mathrm{ABO}$ typing, which are two major factors responsible for $\mathrm{ABO}$ discrepancies in patients who require repeated transfusions. The $\mathrm{ABO}$ distribution among blood donors may well represent that in normal population in that area. Furthermore, another important factor responsible for the difficulty in performing ABO subgroup frequency studies is the very low frequency thereof; thus, extremely large sample sizes are needed. 
With this in mind, the aims of the present study were to determine the incidence of serological $A B O$ subgroups from a large-scale database and describe the features of blood samples with serological ABO discrepancies.

\section{MATERIALS AND METHODS}

\section{Sample collection}

One million samples with available $\mathrm{ABO}$ results were randomly selected from the blood donor database from the Beijing Red Cross Blood Center between 2009 and 2010. Duplicate results were excluded. Furthermore, while the database included Rhesus D-negative individuals, specimens with blood transmitted diseases or that failed to meet the standards of blood donation were excluded.

\section{Serological reverse and forward $\mathrm{ABO}$ typing}

Anti-A, anti-B, and anti-A,B reagents (Beckman Coulter, USA) were used in the determination of the forward $\mathrm{ABO}$ blood group by determining the absence or presence of erythrocytic antigens $\mathrm{A}$ and/or B on the surface of human red blood cells. A cell, B cell, and $\mathrm{O}$ cell reagents (Beckman Coulter, USA) were used to type the reverse $\mathrm{ABO}$ blood groups. A control reagent was used in parallel testing with the blood grouping and phenotyping reagents to differentiate between specific and non-specific agglutination. For the serological typing, an automatic analyzer was used (Beckman Coulter PK7300 System; Beckman Coulter, USA).

\section{ABO subgroup identification}

Further serological tests, including the forward test with anti-Al, anti-H, and anti-A,B; direct agglutinin test; antibody screen test; antibody identification if the antibody screen test was positive; and absorption elution test if the direct agglutinin test was positive, were conducted in all $\mathrm{ABO}$ subgroup samples. All tests followed the standard operation procedures from the Blood Group Lab, Beijing Red Cross Blood Center.
Analysis of the ABO exon 6, 7 sequence and genotyping (Inno-Train Diagnostik GmbH, Germany) were used to confirm cases of $\mathrm{B}(\mathrm{A})$ and cisAB with other subgroups. DNA extraction(QIAamp DNA Blood Mini Kit; Qiagen GmbH, Germany) was conducted using the column extract method.

\section{Statistical analysis}

The frequencies and constituent ratios of the serological $\mathrm{ABO}$ normal types and subgroups were analyzed. The equilibrium between the corresponding normal $\mathrm{ABO}$ types and subgroups was used to confirm the incidence of the A and B subgroups. The odds ratio (OR) with $95 \%$ confidence interval $(\mathrm{CI})$ was used to calculate the hazards for subgroups occurring among types $\mathrm{A}, \mathrm{B}$, and $\mathrm{AB}$. The chi-square test was used to compare the characteristics between the study groups, with a $2-$ sided $P$ value $<0.05$ considered statistically significant.

\section{RESULTS}

\section{ABO distributions for the normal type and subgroups}

Normal ABO types accounted for $99.95 \%$ of the samples (Table 1). Among all normal ABO types, the frequency of the B type was the highest $(31.57 \%$; constituent ratio of the normal type, $31.68 \%)$, followed by $\mathrm{O}(30.56 \%$; constituent ratio, $30.68 \%)$, A (27.28\%; constituent ratio, $27.39 \%)$, and $\mathrm{AB}(10.16 \%$; constituent ratio, $10.19 \%)$. The overall frequency of $\mathrm{ABO}$ subgroups was $0.047 \%$, with AsubgroupB being the most frequently observed subgroup $(0.0288 \%$; constituent ratio of the subgroups, $57.53 \%)$, followed by the B subgroup $(0.0073 \%$, constituent ratio, $14.48 \%), \mathrm{AB}$ subgroup $(0.0058 \%$; constituent ratio, $11.55 \%)$, A subgroup $(0.0056 \%$; constituent ratio, $11.15 \%)$, and others, including para-Bombay, $\mathrm{B}(\mathrm{A})$, and cis $\mathrm{AB}(0.0026 \%$; constituent ratio, $5.29 \%$ ).

\section{Frequencies of serological ABO subgroups}

Table 1 ABO distribution and incidence of subgroup in one million blood donors

\begin{tabular}{|c|c|c|c|c|c|}
\hline \multirow{2}{*}{ ABO group } & \multicolumn{2}{|c|}{ Normal ABO distribution $(99.950 \%)$} & \multirow{2}{*}{ Subgroup } & \multicolumn{2}{|c|}{ ABO subgroup $(0.047 \%)$} \\
\hline & Frequency/\% & Constituent rate $/ \%$ & & Frequency $/ \%$ & Constituent rate/\% \\
\hline A & 27.28 & 27.39 & Asub & 0.0056 & 11.15 \\
\hline B & 31.57 & 31.68 & Bsub & 0.0073 & 14.48 \\
\hline $\mathrm{O}$ & 30.56 & 30.68 & & & \\
\hline \multirow[t]{3}{*}{$\mathrm{AB}$} & 10.16 & 10.19 & AsubB & 0.0288 & 57.53 \\
\hline & & & ABsub & 0.0058 & 11.55 \\
\hline & & & Other & $0.0026^{\circ}$ & 5.29 \\
\hline
\end{tabular}

Including para-Bmbay, cis $\mathrm{AB}$ and $\mathrm{B}(\mathrm{A})$. 
The constituent ratios of the serological $\mathrm{ABO}$ subgroups $(0.047 \%)$ were as follows (Table 2): $7.05 \%$, $47.36 \%, 10.96 \%, 9.00 \%$, and $3.91 \%$ for $\mathrm{A} 2$, A2B, B3, $\mathrm{AB} 3$, and $\mathrm{B}(\mathrm{A})$, respectively. There were 6 types of the A subgroup (A2, A3, Ax, Am, Aint and Aend) and 6 types of A subgroup B (A2B, A3B, AxB, AintB, AendB and $\mathrm{AelB}$ ) in the A subgroup, 5 types of the $\mathrm{B}$ subgroup (B2, B3, Bx, Bm and $\mathrm{Bel})$ and 4 types of the $\mathrm{AB}$ subgroup ( $\mathrm{AB} 3, \mathrm{ABx}, \mathrm{ABel}, \mathrm{ABend})$ in the $\mathrm{B}$ subgroup, other subgroups including $B(A)$, cis $A B$, and para-Bombay $\mathrm{AB}$.

Table 2 Frequencies of serological ABO subgrouptyping in blood donors

\begin{tabular}{|c|c|c|c|c|}
\hline Subgroup & A or B sub & Frequency $/ \%$ & AsubB/ABsub & Frequency/\% \\
\hline \multirow[t]{7}{*}{ Asub } & $\mathrm{A} 2$ & 7.05 & $\mathrm{~A} 2 \mathrm{~B}$ & 47.36 \\
\hline & A3 & 1.76 & A3B & 8.61 \\
\hline & $\mathrm{Ax}$ & 1.37 & $\mathrm{AxB}$ & 0.20 \\
\hline & Am & 0.59 & & \\
\hline & Aint & 0.20 & AintB & 0.98 \\
\hline & Aend & 0.20 & AendB & 0.20 \\
\hline & & & AelB & 0.20 \\
\hline \multirow[t]{6}{*}{ Bsub } & B2 & 1.57 & & \\
\hline & B3 & 10.96 & AB3 & 9.00 \\
\hline & $\mathrm{Bx}$ & 0.98 & $\mathrm{ABx}$ & 1.76 \\
\hline & $\mathrm{Bm}$ & 0.59 & & \\
\hline & Bel & 0.39 & $\mathrm{ABel}$ & 0.59 \\
\hline & & & ABend & 0.20 \\
\hline \multirow[t]{3}{*}{ Other } & $\mathrm{B}(\mathrm{A})$ & 3.91 & & \\
\hline & cis $\mathrm{AB}$ & 1.17 & & \\
\hline & $\mathrm{AB}^{\mathrm{h}}$ & 0.20 & & \\
\hline
\end{tabular}

\section{Equilibrium of $\mathrm{ABO}$ distribution}

We used the risk of the $\mathrm{AB}$ subgroup, including the $\mathrm{A}, \mathrm{B}$ and $\mathrm{AB}$ subgroup, among the normal $\mathrm{AB}$ type as the reference for calculating the odds of the A subgroup among the normal A type and the B subgroup among the normal $\mathrm{B}$ type. Compared to the risk of the $\mathrm{AB}$ subtype, the ORs were $0.06(95 \% \mathrm{CI}$ : $0.05-0.08)$ for the A subgroup and 0.07 (95\%CI: 0.05-0.08) for the B subgroup (Table 3), indicating that the risk of subgroups occurring in people with the $\mathrm{AB}$ type was 14-17-fold higher than that in those with $\mathrm{ABO}$ type $\mathrm{A}$ or $\mathrm{B}$.

\section{DISCUSSION}

This paper reported the distributions of normal $\mathrm{ABO}$ blood types and 14 types of subgroups, along

Table 3 The equilibrium between normal types and subtypes

\begin{tabular}{cccccc}
\hline ABO group & Normal type/\% & Subtype/\% & OR & $95 \%$ CI & $P$ \\
\hline AB & 10.24 & 0.0346 & - & - & - \\
A & 27.38 & 0.0056 & 0.06 & $0.05-0.08$ & $<0.0001$ \\
B & 31.67 & 0.0073 & 0.07 & $0.05-0.08$ & $<0.0001$ \\
\hline
\end{tabular}

with their respective constituent ratios, among one million blood donors in Beijing. These results may offer reference values for studies investigating the associations between the $\mathrm{ABO}$ types and various diseases as a control group from northeastern China, and the data collected may help in establishing strategies related to blood donation or transplantation. Many hospitals in China are currently planning to or have recently established molecular laboratories for transfusion medicine, and our subgroup data may thus also allow labs from different areas to choose the major examination items.

As mentioned above, several case reports focusing on patients with $\mathrm{ABO}$ subgroups have been published, whereas studies on the incidences of $\mathrm{ABO}$ subgroups in the normal population are scarce. Recent studies have demonstrated that the incidences of ABO subgroups were $0.014 \%$ among 190,960 blood donors in Fuzhou ${ }^{[7]}, 0.015 \%$ among blood donors in Dongguan $^{[3]}, 0.016 \%$ among 39,106 blood donors in Pan$\mathrm{yu}^{[9]}$, and $0.024 \%$ among blood donors in Nanning ${ }^{[10]}$, all of which are located in the southeast of China. Similar to our study, the A2 subgroup was the most prevalent $\mathrm{ABO}$ subgroup in these previous studies, indicating that this is the most common subgroup both in the north and south of China. On the other hand, para-Bombay showed an area-specific prevalence in the southeast of China, whereas B(A) was only identified in northern China.

Exact serological ABO typing is the most important examination in transfusion medicine, owing to the risk of severe hemolytic reactions induced by anti-A and anti- $\mathrm{B}^{[11]}$. However, differences between the ABO genotyping and serotyping results may exist, because some subgroups present with the same serotype, such as $\mathrm{Ael} / \mathrm{Bel}$ and $\mathrm{O}$ with the forward typing-negative serotype, and several cases of A205 have moreover been reported to present the same serotyping panel as $\mathrm{Al}$ with anti-A4+ ${ }^{[12]}$. The present study showed that, among others, A205, A207, A208, and A209 reacted with anti-Al, although the guidelines from the State Food and Drug Administration mention that anti-Al cannot react with $\mathrm{A} 2$ and that the agglutinin level of anti-Al with $\mathrm{A} 2$ cells should be as strong as that of A cells ${ }^{[6.12]}$. Due to the limited number of large-scale studies on $\mathrm{ABO}$ genotyping and $\mathrm{ABO}$ sequencing in China, further molecular studies to analyze the $\mathrm{ABO}$ genotype and compare its concordance to the results of serological ABO typing may result in the development of useful tools to more easily determine the presence of the $\mathrm{ABO}$ subtypes and ensure safe transfusions. Although the incidence is low in China, with only approximately $5 / 10,000$ individuals having an 
ABO subgroup in Beijing, the department of transfusion in each hospital should establish a standard procedure for these rare cases. Currently, in Beijing, the Beijing Red Cross Blood Center solves more than 400 cases of suspected $\mathrm{ABO}$ subgroups each year.

In conclusion, the present study analyzed a largescale sample of serological $\mathrm{ABO}$ typing results in one million healthy blood donors in Beijing in order to demonstrate the incidence of $\mathrm{ABO}$ subgroups in this population $(0.0047 \%)$. The $\mathrm{ABO}$ distribution showed that B was the most common normal type, accounting for $31.57 \%$ of cases, followed by $\mathrm{O}(30.56 \%)$, A $(27.28 \%)$, and $\mathrm{AB}(10.16 \%)$. Among the different serological $\mathrm{ABO}$ subtypes, the $\mathrm{A} 2$ subtype showed the highest frequency.

\section{References}

[1] Carnahan RM, Kee VR. A systematic review of validated methods for identifying transfusion-related $\mathrm{ABO}$ incompatibility reactions using administrative and claims data. Pharmacoepidemiol Drug Saf, 2012;21(Suppl 1):230-5.

[2] Makroo RN, Nayak S, Chowdhry M. ABO incompatible renal transplant: Transfusion medicine perspective. Asian J Transfus Sci, 2017;11(1):45-9.

[3] Malhotra S, Dhawan HK, Jain A, et al. Acute Hemolytic transfusion reaction in a patient with Bombay phenotype: Implications for ABO grouping. Indian J Hematol Blood Transfus, 2014;30(Suppl 1):108-10.

[4] Yan L, Zhu F, Xu X, et al. Molecular basis for para-
Bombay phenotypes in Chinese persons, including a novel nonfunctional FUT1 allele. Transfusion, 2005;45(5):725-30.

[5] Chen Q, Li J, Xiao J, et al. Molecular genetic analysis and structure model of a rare $\mathrm{B}(\mathrm{A}) 02$ subgroup of the ABO blood group system. Transfus Apher Sci, 2014;51(2):203-8.

[6] Hua Z, Li L, Li Z. Serological characteristic and molecular basis of A2 subgroup in Shanghai population. Zhonghua Yi Xue Yi Chuan Xue Za Zhi, 2014;31(5):65962.

[7] Chi Q, Zhang A, Ren B. Distribution and molecular genetic analysis of $\mathrm{ABO}$ subtypes in blood donors in Fuzhou area. Chin J Blood Transfusion(In Chinese), 2013; 26(11): 1105-7.

[8] $\mathrm{Hu} \mathrm{Y}, \mathrm{He} \mathrm{Z}$, Chen J. Investigation of ABO subtype and weak D blood donors in Dongguan. J Clin Hematol (In Chinese), 2014; 27(6): 475-7.

[9] Xie J, Deng S, Lan W. Genotypic study of ABO subtypes in Panyu area. Chin J Blood Transfusion(In Chinese), 2016; 29(4): 381-4.

[10] Yan X, Mo Q. Investigation on the distribution of ABO and $\mathrm{Rh}$ blood group of voluntary blood donors in Nanning. Guangxi Medical Journal (In Chinese), 2009; 31(8): 1199-200.

[11] Muller JY, Chiaroni J, Garraud O. Immunological safety of transfusion. Presse Med, 2015;44(2):200-13.

[12] Ying Y, Hong X, Xu X. Serological characteristic and molecular basis of A2 subgroup in the Chinese population. Transfus Apher Sci, 2013;48(1):67-74.

(Received 21 April 2017, Revised 05 May 2017, Accepted 18 May 2017) 